\title{
Systemic or intra-amygdala injection of a benzodiazepine (midazolam) impairs extinction but spares re-extinction of conditioned fear responses
}

\author{
Genevra Hart, ${ }^{1}$ Justin A. Harris, ${ }^{2}$ and R. Frederick Westbrook ${ }^{1,3}$ \\ ${ }^{1}$ University of New South Wales, Sydney New South Wales 2052, Australia; ${ }^{2}$ University of Sydney, \\ Sydney New South Wales 2006, Australia
}

\begin{abstract}
Rats were subjected to one or two cycles of fear conditioning and extinction, injected with a benzodiazepine, midazolam, before the first or second extinction, and tested for long-term inhibition of fear responses (freezing). In Experiment 1, inhibition of context-conditioned fear was spared when midazolam was injected before the second extinction, but impaired when injected before the first. In Experiment 2, it was spared when midazolam was injected before the second extinction, but only if vehicle had been injected before the first: Inhibition was impaired when the drug was injected before both. In Experiment 3, inhibition of a discrete conditioned stimulus (CS A) was spared when midazolam was injected before its second extinction, but impaired when injected before extinction of CS A in rats that had undergone extinction of CS B. In Experiment 4, inhibition was spared when midazolam was injected into the basolateral amygdala before the second extinction, but impaired when injected before the first extinction of context-conditioned fear. The results show that midazolam impairs learning, but not relearning to inhibit fear responses, and are discussed in terms of state dependency, error correction, and memory retrieval, whereby the drug's anxiolytic effects on the second extinction reactivate and strengthen the original inhibitory memory.
\end{abstract}

Clinical trials have established the effectiveness of cognitivebehavioral therapy for anxiety disorders such as posttraumatic stress (Harvey et al. 2003). A component of this therapy is cue exposure in which the patient, aided by the clinician, confronts trauma-related cues in the absence of overt danger. One aim of these confrontations is to reduce, even eliminate, the ability of the trauma-related cues to elicit the fear memories that are undermining the quality of the patient's life. This aim is frequently achieved. However, the psychological processes and neural mechanisms that underlie the successes of cue exposure or that are responsible for its failures are poorly understood. Therefore, there has been much interest in laboratory models of cue exposure. One model is the extinction of Pavlovian conditioned fear responses. Extinction occurs when the signaling relation between a place or stimulus and a feared outcome (e.g., aversive foot shock) is broken by exposures to the now conditioned place or stimulus (CS) in the absence of the aversive unconditioned stimulus (US). The fear responses (e.g., freezing, potentiated startle, analgesia) produced by the excitatory association formed between the CS and US at conditioning decline across CS-alone exposures, and eventually cease to occur. Fear responding to the CS is said to be extinguished.

An historically influential explanation of extinction was that CS-alone exposures erased the learning produced by conditioning (Estes 1955; Rescorla and Wagner 1972). However, it is now clear that much, if not all of this learning survives extinction in spite of the fact that the CS fails to elicit fear responses. This implies that extinction produces new learning that co-exists with the old while inhibiting its expression in fear responses. Moreover, extinction does not just produce inhibitory learning: It also generates a hierarchical organization whereby the physical, temporal, and internal cues, as well as the emotional state present at the time of

\footnotetext{
${ }^{3}$ Corresponding author.
}

E-mail f.westbrook@unsw.edu.au; fax 61-92-03853044.

Article is online at http://www.learnmem.org/cgi/doi/10.1101//m.1154409. extinction come to activate the inhibitory association that opposes the conditioned fear responses (see Bouton 1993). Thus, the fear responses that are inhibited when the CS is subsequently tested in the presence of these cues are restored when it is tested in their absence. For instance, fear responses are "renewed" when the CS is tested either outside of the context where extinction occurred (Bouton and King 1983; Bouton and Ricker 1994; Harris et al. 2000) or in the absence of drug-related cues present during extinction (Bouton et al. 1990); they recover "spontaneously" with the elapse of time, but this recovery is attenuated when the test is preceded by a cue presented during extinction (Brooks and Bouton 1993) and they are "reinstated" when the extinguished CS is tested either in (Bouton and Bolles 1979), or shortly after exposure to (Morris et al. 2005) a dangerous context. Just as extinction leaves intact the excitatory association produced by conditioning, recent evidence also shows that reconditioning an extinguished CS leaves intact the inhibitory association produced by extinction. For instance, such a CS continues to exhibit the signature phenomena of extinction such as spontaneous recovery (Rescorla 2001; Leung and Westbrook 2008). This implies that the learning that produces these phenomena survives reconditioning in spite of the fact that the CS now elicits fear responses (see Rescorla [2001], for discussion).

As noted previously, danger reinstates fear responses to an extinguished CS: It disambiguates the "meaning" of the CS, converting it from one that is safe to one that again elicits fear. In a similar manner, the absence of fear may disambiguate the meaning of a CS that has been extinguished and again conditioned. Specifically, the absence of fear in the presence of such a CS across its second extinction should convert it from one that is dangerous to one that is again safe. The amygdala is a critical component of the neural circuits underlying the acquisition and expression of fear responses. Hence, manipulations that disrupt neural transmission in the amygdala depress fear responding during initial and second extinction. Such a depression could be expected to impair the development of inhibition produced by 
initial extinction if subjects must show fear responses in order to learn to inhibit them (but, see Falls et al. 1992). However, a depression of fear responses across the second extinction could serve to reactivate, and thereby strengthen the inhibitory memory produced by the initial extinction. Support for these suggestions has been provided by demonstrations that disruption of neural activity in the amygdala impairs learning produced by extinction but spares relearning to inhibit fear responses. Specifically, rats that received an intra-amygdala infusion of the anesthetic, bupivacaine (Kim and Richardson 2008), the GABA-A agonist, muscimol, or the NMDA receptor (NMDAr) antagonist, DL-APV (Laurent et al. 2008), showed reduced fear responses across extinction, but substantial levels of such responses on a subsequent drug-free retention test. Rats that had been conditioned, extinguished, again conditioned, and that received these infusions before re-extinction also showed reduced fear responses. However, these rats subsequently showed just as few fear responses on a subsequent test as rats re-extinguished under an infusion of saline into the amygdala.

The present experiments provided a further study of the role of fear responses in learning and relearning inhibition. Rats received two cycles of context-fear conditioning and fear extinction or two context conditioning episodes, followed by initial extinction. A subsequent retention test assessed the long-term suppression of fear responses produced by re-extinction or extinction. We used a benzodiazepine to reduce fear across re-extinction and extinction. Benzodiazepines alleviate reports of fear and anxiety in people and reduce various indices of fear and anxiety in animal subjects. These effects are likely mediated by facilitation of GABA-A receptor transmission in the amygdala. For instance, intra-amygdala infusion of a benzodiazepine reduces various indices of fear and anxiety in animal subjects (Helmstetter 1993; Harris and Westbrook 1995), while infusion of an antagonist facilitates anxiogenic effects (Sanders and Shekhar 1991). Experiments 1-3 studied the effects of a systemically injected benzodiazepine (midazolam) on the inhibitory learning produced by initial extinction and on that produced by second extinction. Experiment 4 examined these effects when midazolam was infused into the basolateral amygdala (BLA). We reasoned that the reduction in fear produced by the drug would impair learning to inhibit fear responses in extinction, but not relearning this inhibition. We hypothesized that rats that entered the second extinction under the influence of the drug would recall initial extinction and that this recall would convert the context from one that was dangerous to one that was again safe.

\section{Results}

\section{Experiment 1}

Experiment 1 studied the long-term inhibition of fear responses in rats systemically injected with midazolam and subjected to their initial or second extinction of context-conditioned fear. Six groups of rats were used $(n=8)$. A summary of the design for Experiment 1 is shown in Table 1. All groups received context conditioning on Day 1. On Day 2, rats in Groups Re-Ext and Home received extinction. On Day 3, all rats received a second context-conditioning episode. On Day 4, rats received their first (Groups Ext) or second (Groups Re-Ext) extinction. This occurred under vehicle for half of the rats in each group (Group Ext VEH and Group Re-Ext VEH) and under midazolam for the remaining rats in each group (Group Ext MID and Re-Ext MID). Drug or vehicle was administered 10 min prior to the onset of extinction. Rats in Groups Home did not receive any extinction but were injected with either vehicle or midazolam in the colony room. Two hours after extinction, rats were given counterbalanced injections. This was done to ensure
Table 1. Design of Experiment 1

\begin{tabular}{|c|c|c|c|c|c|}
\hline Group & $\begin{array}{c}\text { Day } \\
1\end{array}$ & $\begin{array}{c}\text { Day } \\
2\end{array}$ & $\begin{array}{c}\text { Day } \\
3\end{array}$ & $\begin{array}{c}\text { Day } \\
4\end{array}$ & $\begin{array}{l}\text { Day } 5 \\
\text { (Test) }\end{array}$ \\
\hline Re-Ext MID & $C x+$ & $C x-$ & $\mathrm{Cx}+$ & $C x-(M I D)$ & $C x-$ \\
\hline Re-Ext VEH & $C x+$ & $C x-$ & $C x+$ & CX- (VEH) & $C x-$ \\
\hline Home MID & $C x+$ & $C x-$ & $C x+$ & MID & $C x-$ \\
\hline Home VEH & $C x+$ & $C x-$ & $C x+$ & VEH & $C x-$ \\
\hline Ext MID & $C x+$ & Handle & $C x+$ & $C x-(M I D)$ & $C x-$ \\
\hline Ext VEH & $C x+$ & Handle & $C x+$ & $C X-(V E H)$ & $C x-$ \\
\hline
\end{tabular}

Rats were placed in a context $(C x)$ where they were shocked $(+)$ or where fear was extinguished (-). Rats were injected with midazolam (MID) or its vehicle (VEH) before extinction on Day 4.

that any effects on the subsequent test levels of responding were not due to the drug per se. On Day 5, all rats were tested for $10 \mathrm{~min}$ in the context under extinction.

Figure 1 shows the levels of freezing across extinction on Day 2 (Fig. 1A), extinction/re-extinction on Day 4 (Fig. 1B), and test on Day 5 (Fig. 1C). Conditioning on Day 1 was successful. All rats showed post-shock freezing, indicative of context conditioning (Fanselow 1980). Moreover, rats in Groups Home and Re-Ext showed substantial levels of freezing on Day 2. These levels declined across extinction and there were no differences between the groups $(F \mathrm{~s}<1.0)$. Conditioning on Day 3 restored fear responses among rats in Group Re-Ext VEH as they entered extinction on Day 4 with similar levels of freezing as rats in Group Ext VEH. Rats receiving their initial or second extinction under midazolam (Groups Ext MID and Re-Ext MID) did not differ from each other, but froze more and/or were more immobile than rats in the two vehicle groups, $(F=25.5)$. There was a significant linear trend $(F=42.092)$, indicating that overall levels of freezing decreased across extinction. Significant group $\mathrm{X}$ trend interactions confirmed that responding decreased more rapidly for rats in the two vehicle groups than for rats in the two midazolam groups $(F=$ 6.8), and for rats in the Ext VEH group than for those in the Re-Ext VEH group $(F=9.9)$. In fact, there was little or no decline in responses among the drugged rats, suggesting either that extinction had failed or that the drug had produced sedation rather than freezing.

Test data (Fig. 1C) show that rats who received their initial extinction under vehicle exhibited similar low levels of freezing as rats that received their second extinction under either vehicle or midazolam. In contrast, rats that received their initial extinction under midazolam showed similar high levels of freezing as rats that did not receive the second extinction. Statistical analysis confirmed that rats in Groups Ext MID, Home MID, and Home VEH froze significantly more than those in Groups Ext VEH, ReExt VEH, and Re-Ext MID $(F=13.0)$. There were no significant differences between the levels of freezing among rats in Groups Ext MID, Home MID, and Home VEH or between the levels of freezing among rats in Groups Re-Ext MID, Re-Ext VEH, and Ext VEH $(F s<1.0)$.

\section{Experiment 2}

The previous experiment found that rats that received initial extinction under midazolam (Group Ext MID) froze as much on the subsequent retention test, as did rats not subjected to the second extinction (Groups Home). In contrast, rats who received their second extinction under the drug (Group Re-Ext MID) froze on that test as little as did rats subjected to the initial (Group Ext VEH) or second (Group Re-Ext VEH) extinction under vehicle. The present experiment examined the role of the initial extinction in producing this difference on the retention test between the two groups extinguished or re-extinguished under midazolam. 
A

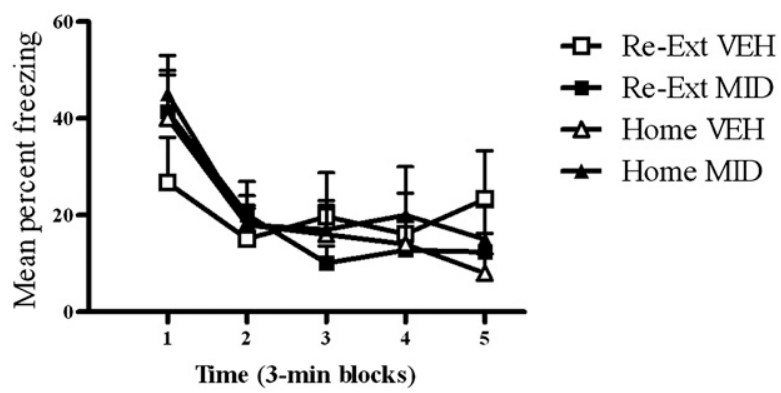

B

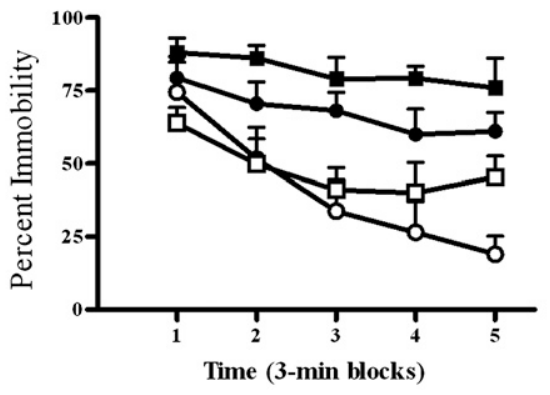

C

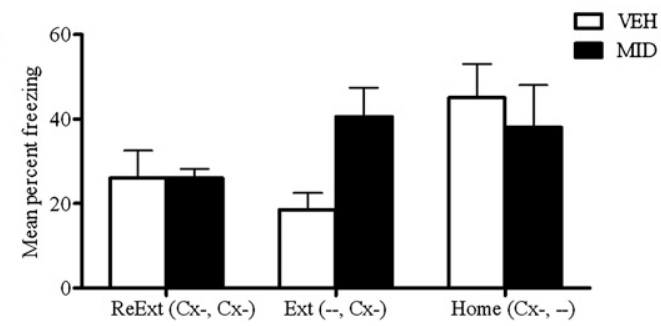

Figure 1. Mean percent (+ SEM) freezing in the conditioned context across extinction $(A)$, extinction/re-extinction $(B)$, and test $(C)$ sessions for rats in each of the groups in Experiment 1. Context conditioning on Day 1 was followed by extinction for rats in Groups Re-Ext and Home on Day 2. Context conditioning on Day 3 was followed by extinction under midazolam (Ext MID) or vehicle or by re-extinction under midazolam (Re-Ext MID) or vehicle on Day 4. Rats in Group Home were injected with drug or vehicle on Day 4. A drug-free retention test was on Day 5.

If midazolam impaired inhibitory learning in initial extinction among rats in Group Ext MID in the previous experiment, then rats in the present experiment that receive their initial extinction under the drug should fail to learn when they receive their second extinction under the drug. Four groups of rats $(n=13)$ were subjected to two cycles of conditioning and extinction, followed by a retention test. The initial experiment $(n=10)$ was replicated with an additional set of rats $(n=3)$ in order to increase power. A summary of the design for Experiment 2 is shown in Table 2. On Day 1, all rats were conditioned to the context. On Day 2, all rats were extinguished to the context, half under midazolam (Groups MID-VEH and MID-MID) and half under vehicle (Groups VEHMID and VEH-VEH). Two hours later, rats were given counterbalanced injections of either drug or vehicle. On Day 3, all rats received a second context-conditioning episode, and, on Day 4, all rats were re-extinguished. Half of the rats originally extinguished under midazolam were re-extinguished under the drug (Group MID-MID); half the rats originally extinguished under vehicle were re-extinguished under the drug (Group VEH-MID), and the remainders were re-extinguished under vehicle (Groups MID-VEH and VEH-VEH). Two hours later, in the colony room, rats received counterbalanced injections of either drug or vehicle to control for any effects of the drug per se on the subsequent test of freezing. The retention test occurred on Day 5.
Figure 2 shows the levels of freezing across the initial extinction on Day 2 (Fig. 2A), the second extinction on Day 4 (Fig. 2B), and test on Day 5 (Fig. 2C). Context conditioning on Day 1 was successful and there were no between-group differences in the levels of post-shock freezing. Across extinction (Fig. 2A), rats extinguished under midazolam (Groups MID-VEH and MID-MID) froze significantly more and/or were more immobile than rats extinguished under vehicle (Groups VEH-VEH and VEH-MID) ( $F=$ 49.2). There were no statistically significant differences between the two MID groups or between the two VEH groups, $F \mathrm{~s}<1.2$. Across the pre-shock period on Day 3, the two groups extinguished under midazolam (Groups MID-MID and MID-VEH) froze significantly more than the two groups extinguished under vehicle (Groups VEH-VEH and VEH-MID) $(F=10.6)$; however, there were no differences between groups post-shock (data not shown). Across re-extinction (Fig. 2B), midazolam-treated rats (Groups MID-MID and VEH-MID) froze significantly more, and/ or were more immobile than the vehicle rats (Groups VEH-VEH and MID-VEH), $(F=26.2)$. There were no significant differences between the two MID groups or between the two VEH groups across this phase. There were significant linear trends across initial extinction on Day 2 and the second extinction on Day 4 ( $F s=25.6$ and 62.6 , respectively), indicating that freezing levels decreased across both.

Test data are presented in Figure 2C. The statistical analysis confirmed what is clear from inspection: Rats that received the initial and second extinction under the drug (Group MID-MID) froze significantly more than rats that received their initial extinction under vehicle but second extinction under midazolam (Group VEH-MID) $(F=18.8)$. There were no statistically significant differences between the remaining two groups (Groups MID-VEH and VEH-VEH) $(F \mathrm{~s}<1.0)$. There was a significant interaction between these two contrasts, indicating that the difference between Groups MID-MID and VEH-MID was significantly larger than the difference between Groups VEH-VEH and MID-VEH ( $F=$ 11.1). Thus, fear responses were reduced on the retention test among rats that received at least one extinction session under vehicle (Groups VEH-VEH, VEH-MID, and MID-VEH) in comparison to the substantial levels of fear responses shown by rats that received both the initial and second extinction under drug (Group MID-MID). Thus, midazolam exerted contrasting effects when administered before the second extinction: Fear responses were low on the subsequent retention test if the initial extinction had occurred under vehicle, but were substantial if the initial extinction had also occurred under the drug.

\section{Experiment 3}

The present experiment provided a further study of the role of the initial extinction in protection against the effect of the drug when administered before the second extinction. The question addressed was whether the learning produced by extinction of one CS afforded protection when extinction of a second CS

Table 2. Design of Experiment 2

\begin{tabular}{lccccc}
\hline Group & $\begin{array}{c}\text { Day } \\
\mathbf{1}\end{array}$ & $\begin{array}{c}\text { Day } \\
\mathbf{2}\end{array}$ & $\begin{array}{c}\text { Day } \\
\mathbf{3}\end{array}$ & $\begin{array}{c}\text { Day } \\
\mathbf{4}\end{array}$ & $\begin{array}{c}\text { Day 5 } \\
\text { (Test) }\end{array}$ \\
\hline VEH-VEH & Cx+ & Cx- (VEH) & Cx+ & Cx-(VEH) & Cx- \\
VEH-MID & Cx+ & Cx-(VEH) & Cx+ & Cx-(MID) & Cx- \\
MID-VEH & Cx+ & Cx-(MID) & Cx+ & Cx-(VEH) & Cx- \\
MID-MID & Cx+ & Cx-(MID) & Cx+ & Cx-(MID) & Cx- \\
\hline
\end{tabular}

Rats were placed in a context $(\mathrm{Cx})$ where they were shocked $(+)$ or where fear was extinguished (-). They were injected with midazolam (MID) or its vehicle (VEH) before extinction on Days 2 and 4. 
A

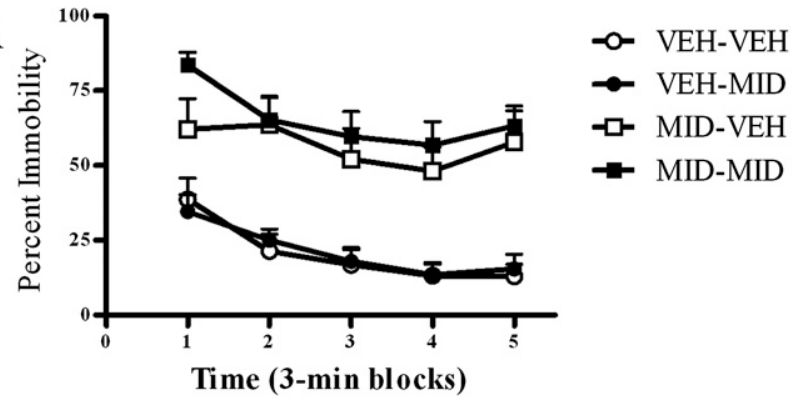

B

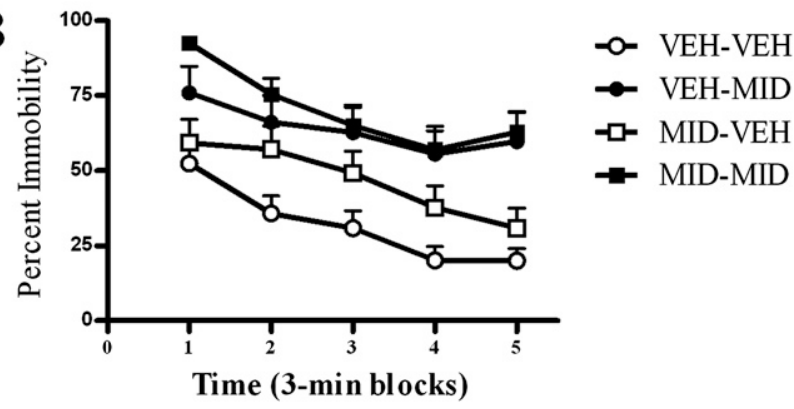

C

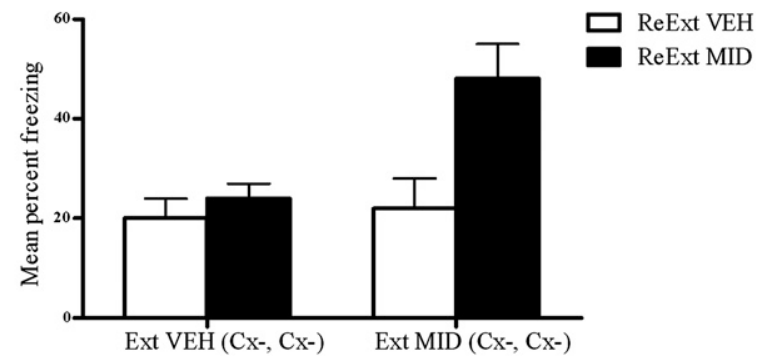

Figure 2. Mean percent (+ SEM) freezing in the conditioned context across extinction $(A)$, re-extinction $(B)$, and test $(C)$ sessions for rats in each of the groups in Experiment 2. Context conditioning on Day 1 was followed by extinction under midazolam (MID-MID and MID-VEH) or vehicle (VEH-VEH and VEH-MID) on Day 2. Context conditioning on Day 3 was followed by re-extinction under midazolam (MID-MID and VEHMID) or vehicle (VEH-VEH and MID-VEH) on Day 4. A drug-free retention test was on Day 5.

occurred under the drug. The design is summarized in Table 3 . It consisted of separately conditioning two discrete CSs (A and B: a flashing light and tone). The stimuli serving as A and B were counterbalanced within each group. Five groups of rats $(n=8)$ were conditioned to each of these CSs on Day 1. On Day 2, three groups were extinguished to CSA (Groups Re-Ext MID, Re-Ext VEH, and Home MID), while two were extinguished to CSB (Groups Ext MID and Ext VEH). On Day 3, all rats were reconditioned to A. On Day 4, two groups received extinction of A under the drug (Groups Re-Ext MID and Ext MID), and two received this extinction under vehicle (Groups Re-Ext VEH and Ext VEH). Rats in Group Home MID were injected with the drug in the colony room but did not receive any other treatment. Two hours later, in the colony room, rats received counterbalanced injections of either vehicle or drug to ensure that any effects on subsequent tests were not due to the drug per se. A retention test of A was conducted on Day 5, drug-free. Thus, A received its second extinction for some rats under the drug and its initial extinction for other rats under the drug.

Figure 3 shows the data across the first extinction on Day 2 (Fig. 3A), the second extinction on Day 4 (Fig. 3B), and test on Day 5 (Fig. 3C). Context conditioning on Day 1 was successful, and there were no between-group differences in the levels of postshock freezing. All rats showed substantial levels of freezing at the onset of extinction (Day 2). These levels decreased across extinction, and there were no differences between groups. Reconditioning on Day 3 restored fear responses to CSA. On Day 4, rats were injected with drug or vehicle and subjected to their initial or second extinction to CSA. Rats injected with the drug froze more and/or were more immobile than rats injected with vehicle across this extinction $(F=46.42)$. There were no statistically significant differences between the two drug groups or between the two vehicle groups. There was a significant linear trend $(F=10.0)$, indicating that overall levels of freezing decreased across the 15-min period.

Test data are presented in Figure 3C. The statistical analysis confirmed what is clear from inspection: Rats that received the initial extinction to CSA under midazolam (Group Ext MID), and those that did not receive a second extinction to CSA (Group Home MID), froze more on the retention test to CSA than those that received their second extinction to CSA under midazolam or vehicle (Groups Re-Ext MID and Re-Ext VEH) or that received their initial extinction to CSA under vehicle (Group Ext VEH) $(F=7.8)$. There were no statistically significant differences among these three latter groups, nor between Groups Ext MID and Home MID $F s<1.0$. Thus, midazolam impaired the long-term inhibition of fear responses when administered before extinction of a CS in rats that had been extinguished to a different CS, but did not impair long-term inhibition when administered before extinction of a CS in rats that had been originally extinguished to that CS. Extinction per se does not protect subsequent extinction from the impairment produced by midazolam.

\section{Experiment 4}

The aim of this experiment was to determine whether the contrasting effects of midazolam on extinction and re-extinction of conditioned fear responses were due to its action in the amygdala. The procedure was similar to that used in Experiment 1; however, rats received an infusion of midazolam or saline into the BLA before extinction (Groups Ext MID and Ext VEH) or reextinction (Groups Re-Ext MID and Re-Ext VEH) of contextconditioned fear responses.

Ten rats were excluded from the statistical analysis because of incorrect cannulae placement or extensive damage to the BLA. Figure 4 shows the location of the cannulae tips for the remaining rats $(n=38)$.

Figure 5 shows the levels of freezing across extinction on Day 2 (Fig. 5A), extinction/re-extinction on Day 4 (Fig. 5B) and test on Day 5 (Fig. 5C). Conditioning on Day 1 was successful. Figure 5A

Table 3. Design of Experiment 3

\begin{tabular}{|c|c|c|c|c|c|}
\hline Group & $\begin{array}{c}\text { Day } \\
1\end{array}$ & $\begin{array}{c}\text { Day } \\
2\end{array}$ & $\begin{array}{c}\text { Day } \\
3\end{array}$ & $\begin{array}{c}\text { Day } \\
4\end{array}$ & $\begin{array}{l}\text { Day } 5 \\
\text { (Test) }\end{array}$ \\
\hline Re-Ext MID & $\begin{array}{l}\mathrm{A}+ \\
\mathrm{B}+\end{array}$ & A- & $\mathrm{A}+$ & $A-(M I D)$ & A- \\
\hline Re-Ext VEH & $\begin{array}{l}\mathrm{A}+ \\
\mathrm{B}+\end{array}$ & A- & $\mathrm{A}+$ & $\mathrm{A}-(\mathrm{VEH})$ & A- \\
\hline Home MID & $\begin{array}{l}\mathrm{A}+ \\
\mathrm{B}+\end{array}$ & A- & A+ & MID & A- \\
\hline Ext MID & $\begin{array}{l}\mathrm{A}+ \\
\mathrm{B}+\end{array}$ & B- & $\mathrm{A}+$ & $A-(M I D)$ & A- \\
\hline Ext VEH & $\begin{array}{l}\mathrm{A}+ \\
\mathrm{B}+\end{array}$ & B- & A+ & $A-(V E H)$ & A- \\
\hline
\end{tabular}

Fear was conditioned (+) or extinguished (-) for two CSs, A and B. Rats were injected with midazolam (MID) or its vehicle (VEH) before extinction on Day 4. 
A

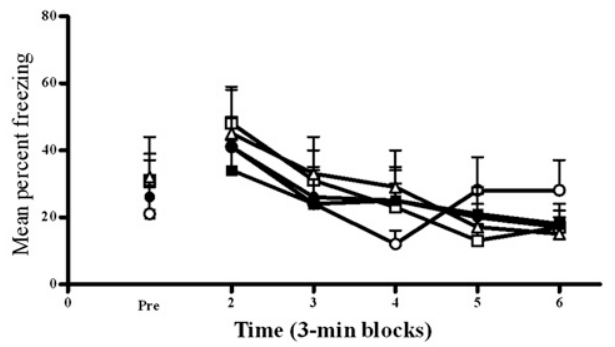

B

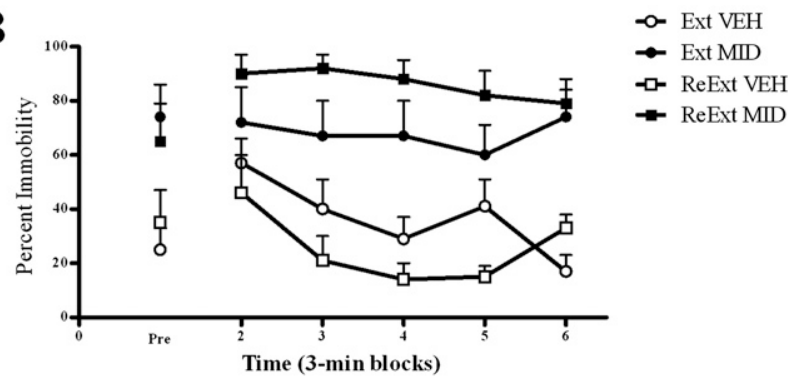

C

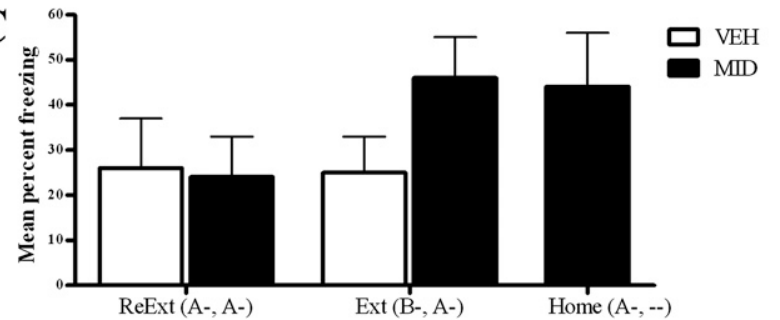

Figure 3. Mean percent (+ SEM) freezing across the extinction $(A)$, reextinction/extinction $(B)$, and test $(C)$ sessions for rats in each of the groups in Experiment 3. Extinction with either CSA (Groups Re-Ext and Groups Home) or CSB (Groups Ext) occurred on Day 2. All groups were reconditioned to CSA on Day 3. Extinction or re-extinction to CSA was conducted under midazolam (Groups Ext MID and Re-Ext MID) or vehicle (Groups Ext VEH and Re-Ext VEH) on Day 4. A drug-free retention test for CSA was on Day 5.

shows that re-exposure to the context on Day 2 (Groups Re-Ext MID [ $n=10]$ and Re-Ext VEH $[n=11]$ ) produced substantial levels of freezing that declined across the session. There was a statistically significant linear trend $(F=22.6)$, confirming loss of fear responses across the session. There were no significant differences between the groups $F<1.0$. The conditioning episode on Day 3 was successful, restoring lost fear responses among rats in Groups Re-Ext. Figure 5B shows that there were substantial levels of freezing by vehicle-treated rats across extinction (Group Ext VEH $[n=9]$ ) and re-extinction (Group Re-Ext VEH) on Day 4. Rats in Groups Ext MID $(n=8)$ and Re-Ext MID froze significantly less than the vehicle rats $(F=11.3)$. Overall, the levels of freezing declined across the session $(F=40.3)$, and this decline was more rapid for rats in the VEH Groups than for rats in Groups MID $(F=$ 8.6). There were no significant differences between the two groups treated with vehicle or between those treated with the drug $(F \mathrm{~s}<$ 1.5).

Test data are presented in Figure 5C. The statistical analysis confirmed that rats subjected to their initial extinction under an infusion of midazolam (Group Ext MID) froze significantly more that rats in the three other groups, $(F=6.7)$. There were no statistically significant differences between the rats re-extinguished under a BLA infusion of midazolam (Group Re-Ext MID) and those extinguished (Group Ext VEH) or re-extinguished (Group Re-Ext VEH) under vehicle $(F s<1.0)$.

\section{Discussion}

The present experiments subjected rats to one or two cycles of fear conditioning and extinction, administered the benzodiazepine, midazolam, before the first or second extinction, and then assessed them drug free for long-term inhibition of fear responses (freezing). Experiment 1 found that the drug impaired long-term inhibition of context-conditioned freezing responses when administered systemically before the first, but not when administered before the second extinction. Experiment 2 showed that the drug spared long-term inhibition when administered before the second extinction, but only when the first extinction had occurred under vehicle: Long-term inhibition was impaired when the drug was administered before the second extinction among rats that had received the first extinction under the drug. Experiment 3

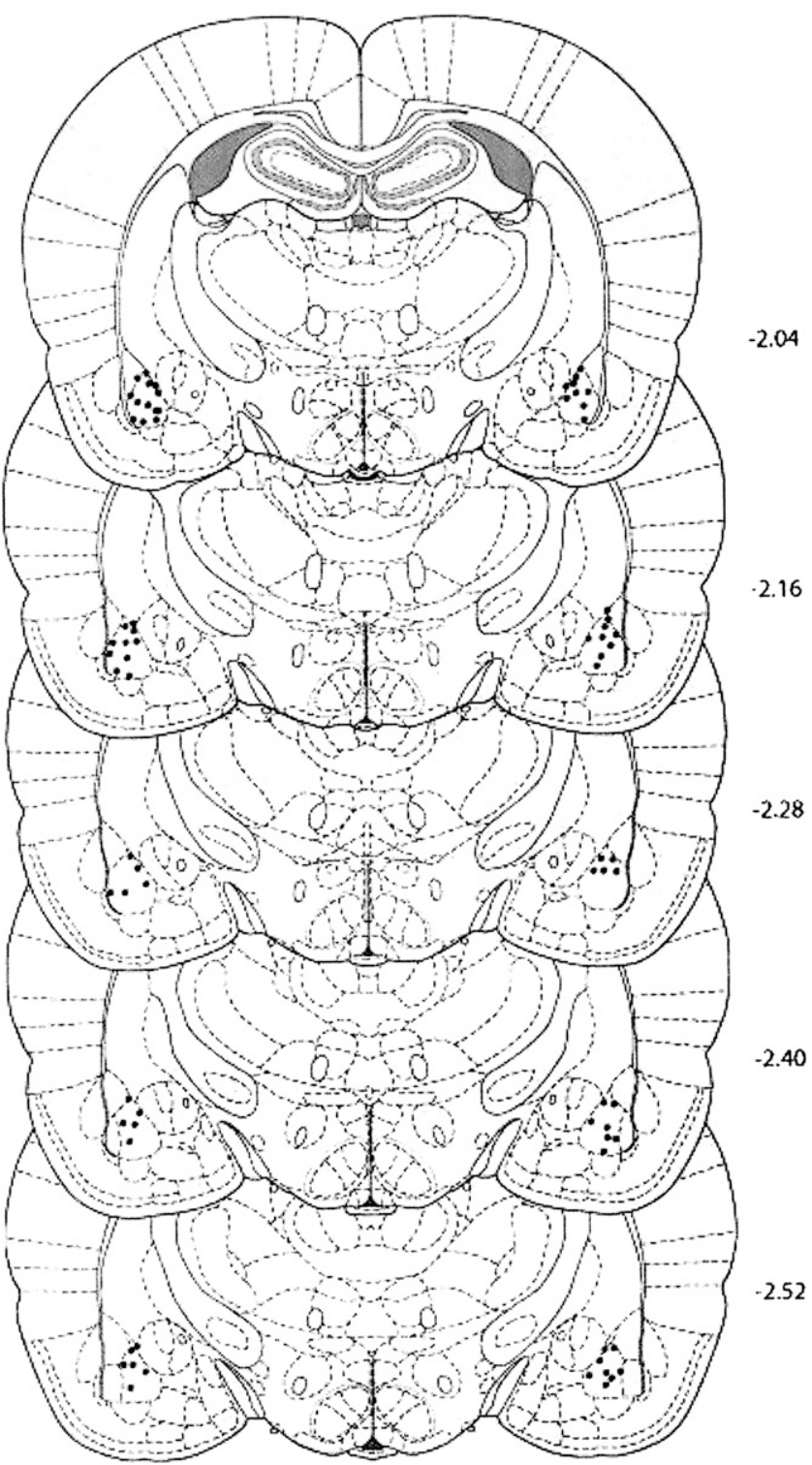

Figure 4. Cannula placement for the remaining 38 rats in Experiment 4. Ten rats in total were not included in the statistical analysis due to cannula misplacement or extensive damage to the BLA. The filled circles represent the location of the cannula tips for the remaining rats at five different rostral-caudal planes. The numbers represent the posterior coordinates (millimeters) from bregma. 
A

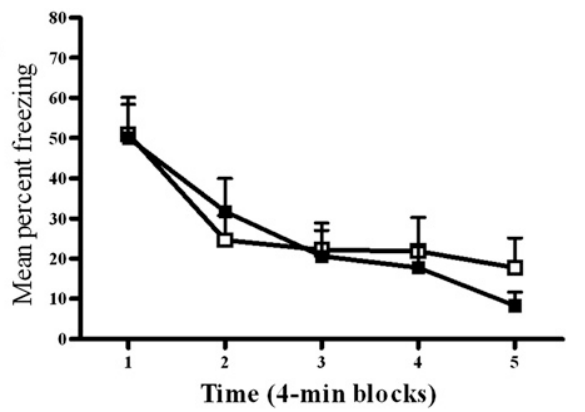

B

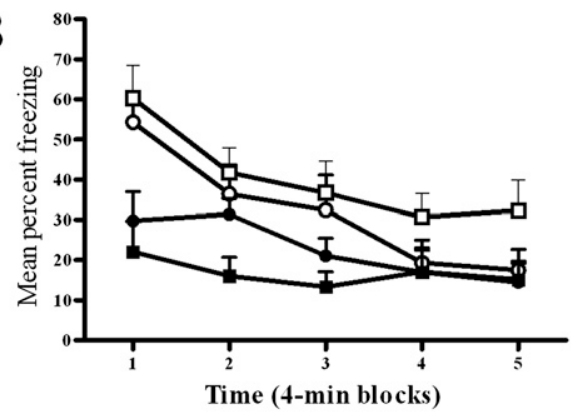

C

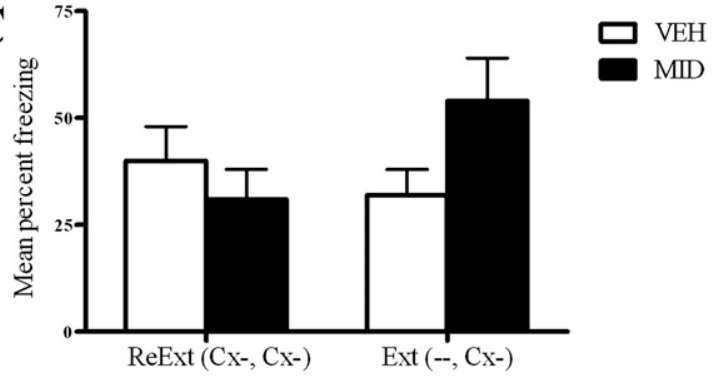

Figure 5. Mean percent (+ SEM) freezing in the conditioning context across extinction $(A)$, re-extinction $(B)$, and test $(C)$ sessions for Experiment 4. Context conditioning on Day 1 was followed by extinction for rats in Groups Re-Ext on Day 2. Context conditioning on Day 3 was followed by extinction under midazolam (Ext MID) or vehicle or by reextinction under midazolam (Re-Ext MID) or vehicle on Day 4. A drug-free retention test was on Day 5.

demonstrated that the drug spared long-term inhibition when administered before the second extinction of a discrete CS (e.g., noise) but only when that (noise) CS had previously been extinguished: When a different (e.g., light) CS had been extinguished, the drug impaired long-term inhibition when administered before subsequent extinction of the noise CS.

Experiments 1-3 failed to detect any direct evidence for anxiolytic properties when the drug was injected systemically. In each of these experiments, rats injected with the drug or saline were immobile or frozen when exposed to the dangerous context or CS at first or second extinction. However, the drug-treated rats remained immobile across the extinction or re-extinction session, whereas saline-treated rats lost this immobility or freezing responses across either session. Experiment 4 provided direct evidence for the anxiolytic properties of the drug. Infusion of the drug into the BLA before the first or second extinction reduced the levels of immobility or freezing relative to saline-treated rats. These results suggest that the failure to detect direct evidence for anxiolytic properties when the drug was injected systemically was due to its sedative effects. Experiment 4 also provided evidence that the long-term effects produced by systemic injection of the drug were mediated by its action in the amygdala. Specifically, the drug impaired long-term inhibition of freezing responses when it was infused into the basolateral amygdala (BLA) before the first extinction, but spared long-term inhibition when infused before the second extinction.

One explanation for the effects of the drug on the long-term inhibition produced by extinction is in terms of state dependency. This holds that drug-related cues constitute the context under which extinction learning occurred. The absence of these cues on the subsequent test resulted in a failure to recall the extinction memory. This failure is an example of the well-documented phenomenon of renewal, whereby the shift of context between extinction and test restores lost fear responses (Bouton and King 1983; Bouton and Ricker 1994; Harris et al. 2000). There is evidence for such an effect, as rats extinguished under a benzodiazepine showed fear responses when tested in the absence of the drug, but no such responses when tested in its presence (Bouton et al. 1990). The problem for this explanation when applied to the results reported here is that drug-related cues did not become part of the context or background under which the second extinction occurred. The subsequent test conducted in the absence of drugrelated cues revealed evidence for recall of the memory formed at the second extinction. This evidence is illustrated by the low levels of fear responses on tests by rats that received their second extinction under the drug (Group Re-Ext MID) versus the substantial levels of test responses by rats treated identically but lacking the second extinction (Groups Home). Moreover, the contrasting effects on the long-term inhibition produced by the first and second extinction were observed when the drug was infused into the BLA, implying that learning, but not relearning inhibition, was under the control of some aspect of the state produced by the BLA infusion.

An alternative description of the present results is that the drug disrupted the learning underlying the long-term inhibition of fear responses, but failed to disrupt relearning this inhibition. A mechanism by which the drug disrupted this learning is its reduction in the levels of activity in the amygdala-based fear system. Contemporary models propose that inhibitory learning in extinction is regulated by the discrepancy between the fear elicited by the CS and the absence of fear appropriate to the new outcome (no shock). Therefore, reducing the levels of activity in the fear system through systemic or intra-amygdala injection of midazolam would reduce this discrepancy and impair the development of inhibitory learning. As noted in the introduction, this inhibitory learning comes under the control of the physical, temporal, and internal cues, as well as the emotional state present at extinction, and its subsequent activation by these cues and state opposes the fear responses elicited by the CS. As was also noted, conditioning an extinguished CS restores fear responses, but does not erase the inhibitory learning produced by extinction. Hence, a CS that had been subjected to two cycles of fear conditioning and extinction entered the second extinction with contrasting memories: The CS that had been rendered safe by the initial extinction is again dangerous as a consequence of its reconditioning. We propose that midazolam disambiguated these memories by reducing the levels of activity in the amygdala-based fear system. This reduction in the levels of activity in the fear system when rats were re-exposed to the dangerous CS in the absence of shock served to return the rats to the emotional state (absence of fear) associated with the initial extinction. This reactivated the inhibitory learning and allowed this learning to be strengthened across the course of the nonshocked re-exposure to the reconditioned context. This strengthening of the original inhibitory learning on the second extinction mediated the absence of fear responses on the subsequent retention test when rats retrieved this inhibitory memory rather than the fear memory. 
Regardless of the underlying mechanisms, the present results obtained with an infusion of a benzodiazepine into the BLA replicate those obtained when the GABA-A agonist, muscimol, the NMDAr antagonist, APV, or the anesthetic, bupivacaine, were infused into the BLA (Kim and Richardson 2008; Laurent and Westbrook 2008; Laurent et al. 2008). Each of these manipulations reduced fear responses across initial and second extinction, each produced impairment in long-term inhibition when infused before initial extinction, but each spared long-term inhibition when infused before the second extinction. The contrasting effect of these manipulations on learning and relearning to inhibit fear responses is at odds with the effect of an intra-BLA infusion of ifenprodil, a selective antagonist of NMRAr containing the NR2B subunit. Rats infused with this drug take longer than controls to suppress fear responses across the initial and second extinction and show substantial levels of fear responding on the subsequent retention tests. Thus, an intra-BLA infusion of ifenprodil disrupts retention of both learning (Sotres-Bayon et al. 2007; Laurent et al. 2008) and relearning (Laurent et al. 2008) to inhibit fear responses. Taken together, these results suggest that exposure to danger activates circuits in the amygdala that trigger the various components of the fear response. Learning or relearning to inhibit the fear response requires activation of NMDAr in the BLA. Consistent with this role of NMDAr activation, disruption of neuronal activity in the BLA impairs inhibitory learning. However, relearning inhibition can occur when neuronal activity in the BLA is disrupted since, as suggested previously, the absence of fear responses reactivates the initial inhibition, which is then strengthened across the course of the second extinction.

Finally, it is worth noting potential implications of the present results for treatment. Firstly, they suggest that treatment of anxiety disorders such as posttraumatic stress with a combination of cue exposure and benzodiazepines will be less effective than cue exposure alone (Birk 2004). However, they also suggest that such a combination will not be less effective than cue exposure alone among patients that have undergone cue exposure and who have suffered a relapse. Indeed, the use of benzodiazepines in combination with cue exposure could even increase compliance, as the anxiolytic effects of the drug will alleviate the fears induced by cue exposure, while not impairing its long-term effectiveness.

\section{Materials and Methods}

\section{Subjects}

Subjects were 188 experimentally naïve male outbred Wistar rats (230-450 g; Rattus norvegicus) obtained from a colony of rats maintained by Gore Hill Research Laboratories. Rats were housed in groups of four to eight in opaque plastic boxes (67-cm depth $\times$ $40-\mathrm{cm}$ width $\times 22-\mathrm{cm}$ height), which were kept in an airconditioned colony room maintained on a 12:12-h light-dark cycle (lights on at 7:00 am). All experiments were conducted during the light portion. Water and standard laboratory chow were continuously available. All experimental procedures were approved of by the Animal Care and Ethics Committee at the University of New South Wales and in accordance with the American Psychological Association guidelines for the treatment of animals in research.

\section{Drug}

In Experiments 1-3, midazolam (Hypnovel, Roche Products) was diluted in sterile isotonic saline $(0.9 \% \mathrm{w} / \mathrm{vol} \mathrm{NaCl})$ to a concentration of $1.25 \mathrm{mg} / \mathrm{mL}$. Midazolam or saline $(0.9 \% \mathrm{w} / \mathrm{vol} \mathrm{NaCl})$ were administered in a volume of $1.0 \mathrm{~mL} / \mathrm{kg}$ via intraperitoneal (ip) injection. Thus, the dose of midazolam was $1.25 \mathrm{mg} / \mathrm{kg}$. This dosage was chosen as one that has been previously used successfully in our laboratory (see Harris and Westbrook 1995, 1998, 1999, 2001).
In Experiment 4, midazolam diluted with saline was prepared to a concentration of $3.3 \mathrm{mg} / \mathrm{mL}$, and the $\mathrm{pH}$ adjusted to 5.0 by adding sodium hydroxide $(\mathrm{NaOH})$. A vehicle solution with the same $\mathrm{pH}$ was also prepared and $0.3 \mu \mathrm{L}$ of midazolam or vehicle solution was infused directly into the basolateral amygdala across $3 \mathrm{~min}$. The infusion cannulae were left for $1 \mathrm{~min}$ more to allow for diffusion.

\section{Surgery and infusion}

Rats were anaesthetized with $(100 \mathrm{mg} / \mathrm{mL})$ ketamine (Ketapex; Apex Laboratories), administered at a dose of $1.0 \mathrm{~mL} / \mathrm{kg}$ (ip) in combination with a muscle relaxant, xylasine $(20 \mathrm{mg} / \mathrm{mL})$, at a dosage of $0.3 \mathrm{~mL} / \mathrm{kg}$ (Rompun; Bayer).

The rat was placed in a stereotaxic apparatus (Kopf Instruments), and two 26-gauge guide cannulae (Plastics One) were implanted through holes drilled in both hemispheres of the skull. The tips of the guide cannulae were aimed bilaterally at the basolateral amygdala located $2.3 \mathrm{~mm}$ posterior to bregma, 4.8-5 $\mathrm{mm}$ lateral to the midline, and $7.7-8 \mathrm{~mm}$ ventral to the skull. The guide cannulae were secured in position with dental cement attached to the skull by three jewelers' screws. A dummy cannula was kept in each guide at all times other than during infusions. Immediately after the surgical procedure, rats were injected ip with a prophylactic $(0.3 \mathrm{~mL})$ dose of $300 \mathrm{mg} / \mathrm{kg}$ solution of procaine penicillin. Rats were allowed $3 \mathrm{~d}$ to recover from surgery, during which time they were handled and weighed daily.

A solution of $0.3 \mu \mathrm{L}$ of midazolam or vehicle was infused bilaterally into the BLA through a 33-gauge internal cannula inserted into the guide cannula. The internal cannula was connected to a $25-\mu \mathrm{L}$ glass syringe attached to an infusion pump (Harvard Apparatus) and projected an additional $1 \mathrm{~mm}$ ventral to the tip of the guide cannula. A total volume of $0.3 \mu \mathrm{L}$ was delivered in both sides at a rate of $0.1 \mu \mathrm{L} / \mathrm{min}$. The internal cannulae were left in place for another $1 \mathrm{~min}$ after the infusions to allow for diffusion. All rats were familiarized with this procedure on the day before infusion by removing the dummy cannulae and running the infusion pump for $3 \mathrm{~min}$.

\section{Histology}

Upon completion of the test, rats were overdosed with pentobarbital, decapitated, and their brains removed. A cryostat was used to slice brains into $40-\mu \mathrm{L}$ coronal sections. Every third section was collected on a slide and stained with cresyl violet. The location of cannula tips was determined under a microscope by two trained observers using the boundaries defined by Paxinos and Watson (1998). Rats with inaccurate cannula placements or with extensive damage to the BLA were excluded from the statistical analysis.

\section{Apparatus}

The experiments took place in a set of four chambers $(23.5-\mathrm{cm}$ depth $\times 20.5-\mathrm{cm}$ width $\times 19.5$-cm height). The sidewalls were constructed of aluminum, while the end walls and lids were constructed of clear Perspex. The floor consisted of stainless-steel bars, $2 \mathrm{~mm}$ in diameter, spaced 13- $\mathrm{mm}$ apart (center to center). Unscrambled AC $50 \mathrm{~Hz}$ shock from a constant current generator could be delivered to the floor of each chamber, and the current available to each floor could be adjusted by reference to an in-line milliampere meter. Below each floor was a tray containing bedding material. Upon removal of a rat, each chamber was cleaned with a paper towel moistened with a $1 \%$ acetic-acid solution, and the bedding changed. Each chamber was located in separate compartments of a wooden cabinet whose floor, ceiling, and walls were painted black. The room containing this cabinet was illuminated by four $40 \mathrm{~W}$ incandescent light bulbs located on the ceiling, two of which were clear white and two red. The background noise level of the room was $50 \mathrm{~dB}$. The behavior of each rat was recorded using a camera mounted on the wall, facing the open compartments. The camera was connected to a video recorder and monitor located in another room in the laboratory. 


\section{Procedure}

\section{Experiment 1}

On Day 1, all rats were placed in the context for $1 \mathrm{~min}$ before receiving a $0.5-\mathrm{mA}, 1-\mathrm{sec}$ foot shock. Rats remained in the chambers for another 2 min before being removed and returned to their home cages. On Day 2, four groups (Groups Re-Ext MID, Re-Ext VEH, Home MID, and Home VEH) received extinction training. This consisted of a 15-min nonreinforced exposure to the context. On Day 3, all rats received identical conditioning as on Day 1, and on Day 4, four groups (Groups Re-Ext MID, Re-Ext VEH, Ext MID, and Ext VEH) received extinction training. Extinction proceeded in an identical manner to that on Day 2; however, 10 min prior to the onset, two groups (Groups Re-Ext MID and Ext MID) were injected with midazolam, and the remaining groups (Groups Re-Ext VEH and Ext VEH) received vehicle. Groups Home MID and Home VEH received injections of either midazolam or vehicle, respectively, in their home cages. Two hours later, all rats received control injections of whichever drug they did not initially receive in order to control for any effects of the drug per se on test performance. On Day 5, all rats were placed in the context for 10 min in the absence of shock and observed for levels of freezing.

\section{Experiment 2}

On Day 1, all rats were placed in the context for 1 min before receiving a $0.5-\mathrm{mA}, 1-\mathrm{sec}$ foot shock. Rats remained in the chambers for another 2 min before being removed and returned to their home cages. On Day 2, all rats received an extinction session, which consisted of a 15-min nonreinforced exposure to the context. This extinction session was preceded by $10 \mathrm{~min}$ by either an injection of midazolam (Groups MID-MID and MIDVEH) or vehicle (Groups VEH-VEH and VEH-MID). All rats received control injections of whichever drug they did not originally receive. On Day 3, all rats received an identical conditioning episode as on Day 1, and on Day 4, all rats received re-extinction. This again consisted of a 15-min nonreinforced exposure to the context, and was preceded by $10 \mathrm{~min}$ by injection of either midazolam (Groups MID-MID and VEH-MID) or vehicle (Groups VEH-VEH and MID-VEH). On Day 5, all rats were placed in the context for $10 \mathrm{~min}$ in the absence of shock and observed for levels of freezing.

\section{Experiment 3}

On the morning of Day 1, rats were placed in the conditioning chamber for $30 \mathrm{sec}$, before a $30-\mathrm{sec}$ presentation of stimulus $\mathrm{A}$ (either a flashing light or a tone, counterbalanced within groups). This stimulus terminated in a $0.5-\mathrm{mA}, 1$-sec shock, and rats remained in the chambers for another 2 min before being removed and returned to their home cages. This procedure was repeated in the afternoon of Day 1 with stimulus B (whichever stimulus rats did not originally receive). On Day 2, three groups (Groups Re-Ext MID, Re-Ext VEH, and Home MID) received extinction of CSA. The remaining groups (Groups Ext MID and Ext VEH) received extinction of CSB. For all groups, extinction consisted of a 3-min adaptation period, followed by a 15-min nonreinforced continuous presentation of the CS. On Day 3, all rats were reconditioned to CSA, which proceeded in an identical way to initial conditioning to CSA on Day 1, and on Day 4, four groups received extinction of CSA. This was identical to extinction on Day 2, except that it was preceded by $10 \mathrm{~min}$ by injection of either midazolam (Groups Ext MID and Re-Ext MID) or vehicle (Ext VEH and Re-Ext VEH). A fifth Group (Home MID) did not receive re-extinction of CSA, but were given an injection of midazolam in their home cages. Two hours after extinction all rats received counterbalanced injections of whichever drug they didn't receive. On Day 5, all rats received a 3-min exposure to the context, followed by a 10-min nonreinforced continuous presentation of the CS, and were observed for levels of freezing.

\section{Experiment 4}

Rats were given $3 \mathrm{~d}$ to recover from surgery, during which they were handled and weighed daily. Context conditioning on Day 1 was preceded by four context pre-exposures ( 2 min per day, for $4 \mathrm{~d}$ ). This pre-exposure has been shown to facilitate the acquisition of contextual fear (Kiernan and Westbrook 1993) and was done in order to minimize potential freezing deficit as a result of surgery. On Day 1, all rats were placed in the context for 1 min before receiving a $0.8-\mathrm{mA}, 1$-sec foot shock. Rats remained in the chambers for a further $2 \mathrm{~min}$ before being removed and returned to their home cages. On Day 2, two groups (Groups Re-Ext MID and Re-Ext VEH) received extinction training. This consisted of a 20-min nonreinforced exposure to the context. On Day 3, all rats received identical conditioning as on Day 1, and on Day 4, all rats received extinction training. Extinction proceeded in an identical manner to that on Day 2; however, immediately prior to the onset, two groups (Groups Re-Ext MID and Ext MID) received BLA infusions of midazolam, and the remaining groups (Groups Re-Ext VEH and Ext VEH) received BLA infusions of vehicle. Six hours later, all rats received control infusions of whichever drug they did not initially receive, in order to control for any effects of the drug per se on test performance. On Day 5, all rats were placed in the context for $10 \mathrm{~min}$ in the absence of shock and observed for levels of freezing.

\section{Scoring and statistical analyses}

Freezing was used to assess conditioned fear. It was defined as the absence of all movement except those related to breathing (Fanselow 1980). Each rat was observed every 2 sec and scored as either "freezing" or "not freezing" by two observers, one of who was blind to group allocation. There was a high degree of agreement between the two observers, with a Pearson product moment correlation $>0.9$. The test data in each experiment were analyzed by a contrast testing procedure that used the Bonferroni inequality to control the experiment wise error rate. Significance was set at $\alpha=0.05$.

\section{Acknowledgments}

This research was supported by grants from Brain Sciences Institute UNSW and the Australian National Health and Medical Research Council.

\section{References}

Birk, L. 2004. Pharmacotherapy for performance anxiety disorders: Occasionally useful but typically contraindicated. J. Clin. Psychol. 60: 867-879.

Bouton, M.E. 1993. Context, time, and memory retrieval in the interference paradigms of Pavlovian learning. Psychol. Bull. 114: 80-99.

Bouton, M.E. and Bolles, R.C. 1979. Role of conditioned contextual stimuli in reinstatement of extinguished fear. J. Exp. Psychol. Anim. Behav. Process. 5: 368-378.

Bouton, M.E. and King, D.A. 1983. Contextual control of the extinction of conditioned fear: Tests for the associative value of the context. J. Exp. Psychol. 9: 248-265.

Bouton, M.E. and Ricker, S.T. 1994. Renewal of extinguished responding in a second context. Anim. Learn. Behav. 22: 317-324.

Bouton, M.E., Kenney, F.A., and Rosengard, C. 1990. State-dependant fear extinction with two benzodiazepine tranquilizers. Behav. Neurosci. 140: $44-55$.

Brooks, D.C. and Bouton, M.E. 1993. A retrieval cue for extinction attenuates spontaneous recovery. J. Exp. Psychol. 19: 77-89.

Estes, W.K. 1955. Statistical theory of distributional phenomena in learning. Psychol. Rev. 62: 369-377.

Falls, W.A., Meserendino, M.J.D., and Davis, M. 1992. Extinction of fearpotentiated startle: Blockade by infusion of an NMDA antagonist into the amygdala. J. Neurosci. 12: 854-863.

Fanselow, M.S. 1980. Conditional and unconditional components of postshock freezing. Pavlov. J. Biol. Sci. 15: 177-182.

Harris, J.A. and Westbrook, R.F. 1995. Midazolam impairs the acquisition of conditioned analgesia if rats are tested with an acute but not a chronic noxious stimulus. Brain Res. Bull. 39: 227-233.

Harris, J.A. and Westbrook, R.F. 1998. Benzodiazepine-induced amnesia in rats: Reinstatement of conditioned performance by noxious stimulation on test. Behav. Neurosci. 112: 183-192.

Harris, J.A. and Westbrook, R.F. 1999. The benzodiazepine midazolam does not impair Pavlovian fear conditioning but regulates when and where fear is expressed. J. Exp. Psychol. 25: 236-246. 
Harris, J.A. and Westbrook, R.F. 2001. Contextual control over the expression of fear in rats conditioned under a benzodiazepine. Psychopharmacology 156: 92-97.

Harris, J.A., Jones, M.L., Bailey, G.K., and Westbrook, R.F. 2000. Contextual control over conditioned responding in an extinction paradigm. J. Exp. Psychol. Anim. Behav. Process. 26: 174-185.

Harvey, A.G., Bryant, R.A., and Tarrier, N. 2003. Cognitive behaviour therapy of posttraumatic stress disorder. Clin. Psychol. Rev. 23: 501-522.

Helmstetter, F.J. 1993. Stress-induced hypoalgesia and defensive freezing are attenuated by application of diazepam to the amygdala. Pharmacol. Biochem. Behav. 44: 433-438.

Kiernan, M.J. and Westbrook, R.F. 1993. Effects of exposure to a to-beshocked environment upon the rat's freezing response: Evidence for facilitation, latent inhibition and perceptual learning. Q. J. Exp. Psychol. $B$ 46B: $271-288$.

Kim, J.H. and Richardson, R. 2008. The effect of temporary amygdala inactivation on extinction and reextinction of fear in the developing rat: Unlearning as a potential mechanism for extinction early in development. J. Neurosci. 28: 1282-1290.

Laurent, V. and Westbrook, R.F. 2008. Distinct contributions of the basolateral amygdala and the medial prefrontal cortex to learning and relearning extinction of context conditioned fear. Learn. Mem. 15: 657-666.

Laurent, V., Marchand, A.R., and Westbrook, R.F. 2008. The basolateral amygdala is necessary for learning but not relearning extinction of context conditioned fear. Learn. Mem. 15: 304-314.
Leung, H.T. and Westbrook, R.F. 2008. Spontaneous recovery of extinguished fear responses deepens their extinction: A role for error-correction mechanisms. J. Exp. Psychol. Anim. Behav. Process. 34: $461-474$.

Morris, R.W., Furlong, T.M., and Westbrook, R.F. 2005. Recent exposure to a dangerous context impairs extinction and reinstates lost fear reactions. J. Exp. Psychol. Anim. Behav. Process. 31: 40-55.

Paxinos, G. and Watson, C. 1998. The rat brain in stereotaxic coordinates, 4th ed. San Diego Academic Press, San Diego, CA.

Rescorla, R.A. 2001. Retraining of extinguished Pavlovian simuli. J. Exp. Psychol. Anim. Behav. Process. 27: 115-124.

Rescorla, R.A. and Wagner, A.R. 1972. A theory of Pavlovian conditioning: Variation in the effectiveness of reinforcement and nonreinforcement. In Classical conditioning II: Current theory and research. (eds. A.H. Black and W.F. Prokasy), pp. 181-215. Appleton-Century-Crofts, New York.

Sanders, S.K. and Shekhar, A. 1991. Blockade of $\mathrm{GABA}_{\mathrm{A}}$ receptors in the region of the anterior basolateral amygdala of rats elicits increases in heart rate and blood pressure. Brain Res. 576: 101-110.

Sotres-Bayon, F., Bush, D.E., and LeDoux, J.E. 2007. Acquisition of fear extinction requires activation of NR2B-containing NMDA receptors in the lateral amygdala. Neuropsychopharmacology 32: 1929-1940.

Received July 12, 2008; accepted in revised form November 2, 2008. 


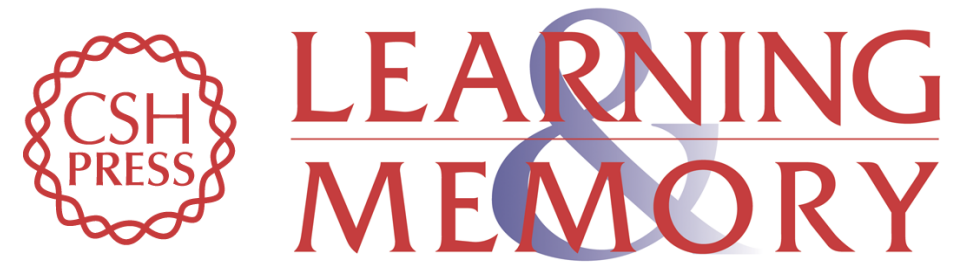

\section{Systemic or intra-amygdala injection of a benzodiazepine (midazolam) impairs extinction but spares re-extinction of conditioned fear responses}

Genevra Hart, Justin A. Harris and R. Frederick Westbrook

Learn. Mem. 2009, 16:

Access the most recent version at doi:10.1101//m.1154409

References This article cites 26 articles, 4 of which can be accessed free at: http://learnmem.cshlp.org/content/16/1/53.full.html\#ref-list-1

License

Email Alerting Receive free email alerts when new articles cite this article - sign up in the box at the Service top right corner of the article or click here. 